\title{
Comparación de dos férulas de distinto material en cuanto a efectividad en amas de casa con Síndrome del Túnel Carpiano ${ }^{1}$
}

\author{
Autoras: Delia Viviana Santaella, T.O.* \\ Helina Margarita Herrera, T.O. **
}

\section{INTRODUCCIÓN}

A través de este trabajo aplican los conocimientos adquiridos en el Diplomado de Férulas en miembro superior, como también nuestros conocimientos como profesionales de la Terapia Ocupacional y Diseño Industrial.

En específico se ha enfocado hacia el Síndrome del Túnel Carpiano (STC) que se presenta frecuentemente en mujeres entre 30 y 35 años con actividad ocupacional, amas de casa y con síntomas iniciales de la patología.

Se analizó la efectividad de la reducción de los síntomas del STC a través de dos férulas de diferente material analizando las siguientes variables:

1. Beneficios (costos - durabilidad)

2. Reducción de síntomas (dolor - hormigueo - parestesias) y

3. Material:

Férula I: Confeccionada en Orthoplas.

Férula II: Confeccionada en Poliuretano de densidad media.

\footnotetext{
1 RECONOCIMIENTO TRABAJO DE INVESTIGACIÓN - XI Congreso Nacional de Terapia Ocupacional. Modalidad: Profesionales.

Este trabajo se elaboró como proyecto investigativo a las condiciones del Diplomado de férulas de miembro superior realizado por la Escuela Colombiana de Rehabilitación.

* Escuela Colombiana de Rehabilitación afiliada al Colegio Mayor de Nuestra Señora del Rosario.

** Escuela Colombiana de Rehabilitación afiliada al Colegio Mayor de Nuestra Señora del Rosario.
} 
Se escogió como base de la muestra la ciudad de San José de Cúcuta, Departamento Norte de Santander, tomando como base la estadística realizada en el Departamento de Terapia Ocupacional del Instituto de los Seguros Sociales de esta ciudad en donde se presenta una alta incidencia de esta enfermedad.

Cabe resaltar que actualmente se viene realizando las Férulas tipo I, es decir usando el material Orthoplast en el tratamiento del síndrome en el estadio temprano, pero que sus altos costos como las molestias causadas por el calor nos han llevado a pensar en la realización de la férula en otro material económico, escogiendo para ello el Poliuretano de densidad media con el cual se confeccionó la Férula tipo II.

\section{ANTECEDENTES}

\subsection{Antecedentes históricos}

\section{Definición:}

El síndrome del Túnel carpiano es una condición inhabilitante que se produce, por la comprensión del nervio mediano a su paso por el túnel carpiano. Es un trastorno relativamente común debido a múltiples causas como trauma, enfermedades predisponentes, estados fisiológicos como embarazo, consumo de alcohol y anticonceptivos orales.

El túnel carpiano es un hueso en forma de corchete en la muñeca, que se ramifica hacia el pulgary los tres primeros dedos, debajo de este Túnel está el nervio mediano y los tendones que controlan los dedos; si los tendones del túnel carpiano se hinchan pueden comprimir el nervio mediano causando una sensación de palpitación o entumecimiento en la mano.

\section{Historia del proyecto}

La Terapeuta Ocupacional Delia Bibiana Santaella Carrero del servicio de rehabilitación, departamento de Terapia Ocupacional, Clínica del Seguro Social, de la Ciudad de San losé de Cúcuta, Norte de Santander viene elaborando una estadística de la incidencia de esta enfermedad analizando básicamente edad, sexo, ocupación, evolución, desde enero de 1997. 
En marzo de 1998, como proyecto de tesis del Diplomado de Férulas de miembro superior, se escogió realizar un estudio basado en pacientes de oficio amas de casa, a las cuales se les elaboró férulas en dos tipos de materiales, para realizar una comparación y analizar variables como beneficios y efectividad.

\subsection{Datos estadísticos}

La mayoría de los casos de los síntomas del síndrome del túnel carpiano aparecen espontáneamente.

Existen ciertos factores predisponentes tales como la comprensión y estiramiento crónico ocupacional del nervio mediano por flexión o extensión repetitiva de la muñeca y por la estreches del túnel. Su comienzo es espontánea y gradualmente progresivo.

La incidencia es más común en mujeres que en hombres $(2: 1)$ y en ambos casos especialmente en la edad media. La mano dominante es la más comprometida, teniendo en cuenta que puede ser bilateral.

El Síndrome del Túnel Carpiano puede ser asociado con la enfermedad reumática, secundario de la fractura de coollex, puede también darse en una variedad de condiciones médicas como es el embarazo, diabetes mellitus, enfermedades de la tiroides. Sin embargo la gran mayoría de pacientes con este síndrome no se pueden asociar con una enfermedad sintomática.

La mano generalmente lucirá normal, sin embargo en estadios de evoluciones largas se atrofian los músculos inervádos por el nervio mediano.

Los síntomas se manifiestan como parestesias y dolor en los tres primeros dedos inervados por el nervio mediano, (pulgar-índice-medio). Aunque en anular en cara radial también puede ser comprometido.

Estas parestesias y el dolor se extiende a toda la mano, los brazos y los hombros y junto con la sensación de plenitud de la mano afectada y el dolor son muy severos durante la noche y más al despertar. 
Los síntomas también se presentan frecuentemente cuando el paciente carga objetos, lava, teje, borda, escurre el trapero, o la ropa, escribe en exceso o sostiene objetos en la mano.

\subsection{Estudios anteriores de referencia}

Según Phalen G, en 1970, después de investigar durante 21 años, determinó que la causa de consulta más común por dolor difuso en la mano, era el síndrome del Túnel Carpiano.

Estudios epidemiológicos Norteamericanos y Holandeses han sugerido que un $0.1 \%$ de la población padece del síndromè del Túnel Carpiano y un $4.0 \%$ de los casos se han asociado a causas ocupacionales.

Jetezn y colaboradores encontraron en la escanografía anormalidades de la muñeca en el $78.2 \%$ de los pacientes, encontraste con el $53.1 \%$ de positividad electromiográfica en las mismas.

Richard M. Gelberman y colaboradores han dividido el desarrollo del síndrome del Túnel Carpiano, en cuatro estadios de tal manera que según el estadio en que se encuentra la persona se define el tratamiento. Estos son: Estadio Temprano, Estadio Intermedio, Estadio Avanzado, Estadio Agudo.

\section{JUSTIFICACIÓN}

Los antecedentes nos indican que este síndrome es más frecuente en mujeres en edad media que realizan actividades como cargar objetos, lavar, escurrir el trapero, bordar, etc., actividades prevalentes en las labores de la ama de casa.

De acuerdo a la estadística realizada y en específico a la elaborada en el Departamento de Terapia ocupacional del Seguro Social de San José de Cucutá, se corroboró lo anteriormente expuesto, escogiendo el estadio temprano del síndrome que para su tratamiento utiliza medidas conservadoras de la inmovilización de la muñeca que puede consistir en el uso permanente de una férula que mantenga la muñeca en ligera dorsiflexión ( $10^{\circ}$ a $15^{\circ}$ de extensión). 
Considerando que las férulas comúnmente se realizan con materiales termoplásticos de baja temperatura y de alto costo, para ciudades calientes como San José de Cúcuta el uso de este material ha presentado molestias o incomodidad en el paciente (sudoración excesiva, prurito, y mal olor).

Justificando esta investigación en la búsqueda de una Férula confeccionada en otro tipo de material que reduzca costos y soluciones estas incomodidades en el paciente y disminuya la cantidad de pacientes que por sus altos costos no pueden tener acceso a estas aumentando los síntomas y haciéndose necesario unos tratamientos más costosos y dolorosos.

\section{OBJETIVOS}

\subsection{Objetivo general}

Comparar la efectividad de las férulas confeccionadas en dos tipos de material (Orthoplas Vs. Poliuretano de baja densidad) en el tratamiento del síndrome del Túnel Carpiano (STC) en fase temprana, amas de casa, de edad media en la Ciudad de San José de Cúcuta, Norte de Santander.

\subsection{Objetivos específicos}

Como objetivos específicos se han definido:

- Escoger la muestra apropiada de acuerdo a las historias clínicas teniendo en cuenta, edad, ocupación y estadio de la enfermedad.

- Determinar el tiempo de permanencia de la férula en el paciente y realizar un seguimiento de su evolución.

- Disminución de costos en la elaboración de las férulas.

- Comparar la durabilidad de las férulas según el tipo de material

- Aumentar el número de pacientes que puedan realizar el tratamiento completo y disminuir los síntomas del STC en el inicio o estado temprano de la enfermedad. 


\section{FORMULACIÓN DEL PROBLEMA}

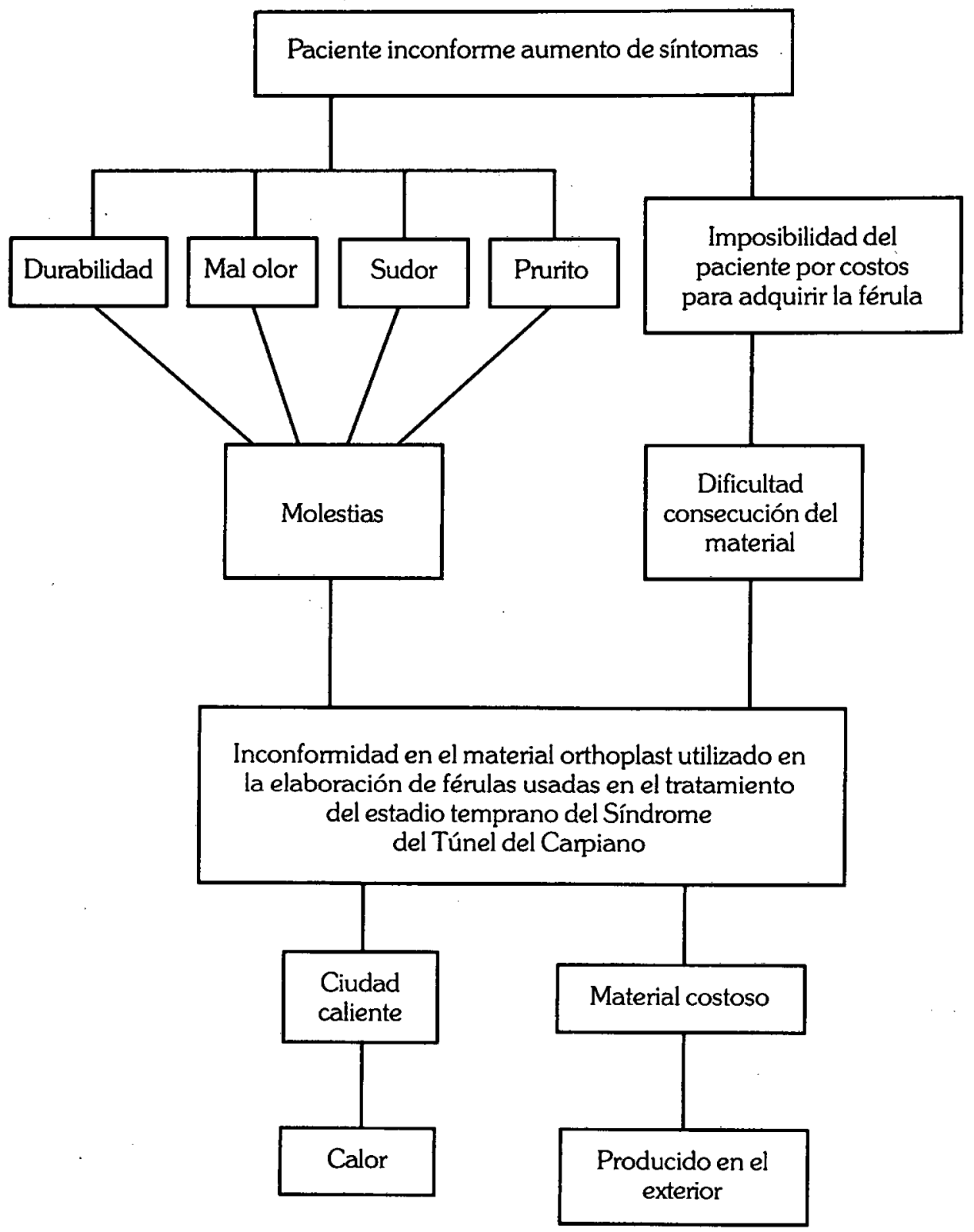

Esquema No. 1 
La inconformidad en el material ORTHOPLAS utilizado en la elaboración de férulas usadas en el tratamiento del Estadio Temprano del Síndrome del Túnel del Carpio, causado específicamente en ciudades calientes (altas temperaturas, excesivo calor) y por los altos costos del material por ser este un producto elaborado en el exterior.

Generándose molestias en el paciente como son malos olores, pruritu, sudoración excesiva y baja durabilidad, como también dificultad de adquirir este material por sus altos costos y con ellos aumentando el número de pacientes que no pueden llevar a cabo el tratamiento en el estadio temprano del síndrome y con ello aumentando los síntomas hasta pasar a los estadios intermedio, avanzado y en el peor de los casos llegando al Síndrome del Túnel Carpiano Agudo, que obligan al paciente a tratamientos más costosos, dolorosos y muchas veces con efectividades bajas.

\section{REVISIÓN LITERARIA}

\subsection{Definición}

El Síndrome del Túnel Carpiano es una comprensión del nervio mediano en la muñeca, en el túnel del carpo. Es la neuropatía por atrapamiento más común en el miembro superior.

\subsection{Etiología}

La mayoría de los casos del STC espontáneamente. Existen ciertos factores predisponentes tales como la comprensión y estiramiento crónico ocupacional del nervio mediano por flexión o extensión repetitiva de la muñeca y por trauma de ella, con fractura o sin ella y con formación de un hematoma o de inflamación. El embarazo al retener líquidos, y las enfermedades sistémicas que conducen a inflamación y edema crónico tales como diabetes, la acromegalía y el mixedema,

pueden ocasionar el estrechamiento del túnel carpiano y con ello los síntomas de comprensión. También pueden ocacionarlo la artritis rematoidea, la sarcoidosis, la amiloidosis, la gota y otras enfermedades inflamatorias sistémicas, las infecciones localizadas en la muñeca como la artritis séptica, la tuberculosis y la lepra. Anomalías congénitas del túnel o de las estructuras contenidas dentro del túnel, 
tales como las arterias aberrantes, hipertrofia de los tendones flexores, o del tendón del palmar mayor y gangliones de los tendones de los flexores de los dedos o del flexor largo del pulgar también pueden ocasionarlo. En la polineuropatía diabética hay una mayor labilidad del paciente a sufrir atrapamientos nerviosos, en especial el Síndrome del Túnel Carpiano.

En los casos en los cuales no existe una causa, el STC resulta del trauma repetitivo sobre el nervio mediano ocasionado por los movimientos de flexoextensión de la muñeca y la estrechez del túnel.

\subsection{Síntomas y signos}

Su comienzo es espontáneo y gradualmente progresivo. Afecta dos veces mas a las mujeres que a los hombres y en ambos casos especialmente en la edad media.

El dolor en los dedos y las parestesias, los cuales afectan especialmente los tres primeros dedos inervados por el nervio mediano, aunque el anular èn su cara radial también puede estar comprometido, son los síntomas más comunes. A veces estas parestesias y el dolor se extienden a toda la mano, los brazos e incluso los hombros pero raramente afectan al meñique. Ellas junto con la sensación de plenitud de la mano afectada y el dolor, son muy severas durante la noche y más al despertar. $\mathrm{El}$ paciente se libera de estas molestias sacudiendo su mano o colgándola sobre el borde de la cama. Los síntomas también se presentan frecuentemente cuando el paciente carga objetos, escribe en exceso, teje, borda, lava, escurre la trapeadora o ropa o sostiene objetos en las manos y pueden durar meses con periodos de exacerbación y remisión.

Al examen se puede encontrar disminución de la sensibilidad en la zona de la inervación correspondiente al mediano en los dedos de la mano, paresia de los músculos tenares, en especial del abductor corto y del oponente del pulgar y la atrofia de estos músculos en caso de evolución larga. Los cambios troficos no son frecuentes. El signo de Tinel, consistente en parestesias en la zona de la distribución cutánea distal al sitio donde se percute un nervio periférico, puede presentarse al percutir al mediano en la muñeca, en la parte proximal de la palma.

La maniobra de Phalen, consiste en hiperflexión o hiperextensión de la muñeca, reproduce las parestesias en los dedos en un $50 \%$ de los casos del Síndrome del Túnel Carpiano. 


\subsection{Diagnóstico}

Se basa en los síntomas descritos por el paciente y los signos positivos al examen clínico. La radiografía de la muñeca puede revelar fracturas antiguas o cambios artríticos o artrósicos que estrechan el túnel del carpo. La escenografía puede revelar una estenosis del canal del carpo, un engrosamiento del ligamento transverso con disminución secundaria del volumen del túnel y una comprensión de su contenido, un engrosamiento de la envoltura sinovial de los tendones flexores, lo cual aumenta el volumen de estos y comprime el nervio mediano y una fibrosis recurrente después de la cirugía. Jetzen y colaboradores encontraron en la escanografía anormalidades de la muñeca en el $78.2 \%$ de los pacientes, en contraste con $53.1 \%$ de positividad electromiográfica en las mismas. Sin embargo, los cambios electrofisiológicos son los únicos que nos dicen si el nervio esta afectado como consecuencia de las alteraciones vistas en la escanografia. Los estudios de resonancia magnética permiten visualizar las estructuras óseas y blandas contenidas dentro del resto de estructuras, al igual que medir su espesor y su anchura.

El diagnostico clínico del síndrome del túnel carpiano se comprueba mediante estudio de neuroconducción del mediano y electromiografia. La primera alteración es el aumento del tiempo de latencia sensitivo al estimular el nervio mediano en la muñeca y captar dostalmente a $14 \mathrm{~cm}$ sobre los dedos índice, medio o anular, y a él le siguen disminución de la amplitud de los potenciales de acción nerviosos sensitivos, aumento del tiempo de latencia motor distal al estimular el nervio mediano de la muñeca y captar distalmente a $8 \mathrm{~cm}$ sobre los músculos tenares, y disminución de la amplitud de los potenciales de acción muscular compuestos. La electromiografia puede mostrar signos de denervación en los músculos oponente y abductor corto del pulgar en casos severos y antiguos, en los cuales se ha producido un daño axonal motor del nervio mediano.

El diagnóstico clínico de atrapamiento del nervio mediano en el túnel del carpo sin resultados positivos electromiográficos es poco común y el tratamiento quirúrgico en general no tiene éxito.

A pesar de que el STC es la causa más común de las parestecias en la mano, otras condiciones deben tenerse en cuenta en el diagnóstico, tales como una polineuropatía, atrapamientos nerviosos más proximales, radiculopatia cervical, síndrome del opérculo torácico y plexopatia del plexo braquial. Otros atrapamientos 
como el síndrome del pronador redondo y del nervio ulnar en el codo puede distinguirse clínicamente y por electrodiagnóstico.

\subsection{Tratamiento}

El tratamiento inicial puede consistir en el uso nocturno de una férula que mantenga la muñeca en ligera dorsiflexión -10 grados-, o en el cambio de ocupación. También se puede hacer infiltraciones del túnel carpiano con esteroides, aunque se corre el riego de incrementar el daño al nervio en caso de efectuarla en forma intraneural.

Richard H. Gelbereman y colaboradores han dividido el desarrollo del STC en cuatro estadios, de tal manera que según el estadio en que se encuentre la persona se define el tratamiento.

- Estadio temprano del síndrome del túnel carpiano. Los pacientes que se encuentran en este estadio son los que han presentado los síntomas por un periodo menor de un año.

- Estadio intermedio del síndrome del túnel carpiano. Este estadio incluye pacientes que tienen interferencia persistente de la microcirculación endoneurial, con edema intrafascicular y epineural, síntomas clínicos de parestesia y entumecimiento constante, valores de discriminación de dos puntos altos y aumento de los tiempos de latencia distales sensitivo y motor del nervio mediano. El tratamiento de elección es la descompresión quirúrgica del túnel carpiano mediante incisión del ligamento transverso del carpo.

- Estadio avanzado del síndrome del túnel carpiano. Incluye a las personas con compresión crónica del nervio mediano que presenta edema epineural por tiempo largo, seguido de edema intrafascicular y endoneurial que interfiere con el transporte axonal y disminuye el flujo capilar. El edema endoneurial prolongado induce a la fibrosis endoneurial y finalmente a la desmielinización y la degeneración axonal. Es probable que una vez que la fibrosis endoneurial y la degeneración axonal hayan ocurrido, la neurolisis no mejore la función del nervio.

- Síndrome del túnel carpiano agudo. La comprensión aguda del nervio mediano en la muñeca es poco frecuente. Se ha observado en hemofilicos que 
sangran, en pacientes anticuagulados, después de trombosis de la arteria mediana, en luxaciones de los huesos del carpo o de la base de los metacarpianos, en fracturas, infecciones y durante el embarazo. Las fracturas distales del radio son la causa más frecuente. En resumen, el tratamiento del síndrome del túnel carpiano mediante su infiltración con esteroides proporciona alivio por algún tiempo, pero los síntomas vuelven a aparecer en un tiempo variable que oscila entre dos meses a un año. Las infiltraciones dentro del nervio o el tendón, a repetición, pueden ocasionar mayor daño al mediano, atrofia y ruptura de los tendones flexores.

\subsection{Estado temprano del Síndrome del Túnel Carpiano}

Los pacientes que se encuentran en este estadio son los que han presentado los síntomas por un periodo menor de un año, entumecimiento difuso e intermitente, discriminación normal de dos puntos, ausencia de paresia muscular y de atrofia. El estudio electrofisiológico de estos pacientes muestra aumento de los tiempos de latencia leves y ausencia de fibrilación.

Para su tratamiento se han utilizado medidas conservadoras como inmovilización de la muñeca, $e$ infiltraciones con esteroides, aunque no se ha logrado probar su eficacia. Los pacientes en estado temprano con un bajo grado de comprensión del nervio mediano responden favorablemente a las medidas conservadoras consisten-

- tes en infiltración del túnel carpiano con una mezcla de $1 \mathrm{ml}$ de esteroide hidrosoluble y $1 \mathrm{ml}$ de xilocaina o bupivacaina al $5 \%$ sin epinefrina, e inmovilización con una férula palmar de la muñeca en posición de dorsiflexión -10 grados- durante tres semanas.

Cuando los síntomas son severos desde el comienzo, hay una recurrencia de éstos después del tratamiento hasta en el $89 \%$ de los casos.

\section{Tratamiento}

La naturaleza del tratamiento conservador depende de la etiología y de la condición del STC. Si un desorden metabólico como la artritís reumatoidea o la diabetis contribuyen a los síntomas del STC, el control médico es una línea primaria de acción. 
Si los síntomas son provocados por una actividad particular, se debe disminuir la duración en la ejecución de la misma o interrumpir el desempeño de la actividad.

En la etapa inicial cuando solo hay dolor y adormecimiento el tratamiento se basa en:

Inmovilización de la muñeca en 10 a 15 grados de extensión (evitando la hipereflexión durante el sueño), prohibiendo la realización de actividades repetitivas que implique sobreesfuerzos y evitar cargar objetos con la mano afectada.

La férula puede ser diseñada en materiales termoplásticos de baja temperatura como el Orthoplast que va palmarmente desde el tercio medio del antebrazo hasta el pliegue discal de la palma de la mano, permitiendo la flexoextensión de las articulaciones metacarpofalángicas e interfalángicas de los dedos de la mano enferma.

Durante esta etapa el paciente puede realizar sus actividades diarias utilizando la férula siempre y cuando se eviten actividades contraproducentes.

Infiltración anestésica con corticoesteroides del túnel carpiano sólo en casos iniciales, al mismo tiempo con el uso de la férula siendo inyectada hasta por tres veces con intervalos no menores de un mes cada una.

\subsection{Férulas}

\subsubsection{Definición}

La férula u ortisis es una fuerza aplicada directa o externamente al cuerpo de un individuo con el objeto de soportar, corregir una deformidad debilitada o anatómica permitiendo o restringiendo un movimiento corporal.

También puede definirse como un aparato rígido o flexible usado para prevención del movimiento de una articulación o para la fijación de desplazamientos o partes móviles.

\subsubsection{Tipos de férulas}

Las férulas cuya función se acopla directamente con la corrección y o prevención de deformidades se clasifican independientemente así: 
Según su función en estáticas y dinámicas. Las férulas estáticas mantienen los segmentos óseos en una posición determinada permitiéndole o no la habilidad de otro. Las férulas dinámicas permiten el libre juego de los segmentos articulares, asiste a la función de músculos debilitados por medio de un accesorio facilitando el movimiento.

Según su nivel cortas y largas. Las férulas cortas corresponden a los segmentos de muñeca, manos y dedos. Y las largas sirven para corregir o prevenir segmentos de hombro, codo, muñeca, antebrazo y mano.

En el tratamiento del Estadio temprano del STC se utiliza una férula estática y corta.

\subsubsection{Características de las férulas}

Una adecuada férula debe cumplir las siguientes características:

- Cumplan los objetivos del tratamiento.

- Sean livianas, sencillas y fácil adaptabilidad.

- Con manufactura estandarizada

- De rápido arreglo y mantenimiento.

- Ajustables, cosméticas, higiénicas, seguras.

- Aceptadas por el paciente.

\subsubsection{Materiales en la elaboración de férulas}

\subsubsection{Material termoplásticos}

Muchos de los materiales básicos utilizados actualmente son termoplásticos de baja temperatura. Con calor estos materiales se vuelven blandos y fléxibles y cuando son enfriados mantienen la forma en que fueron moldeados. Aunque los materiales temoplásticos difieren en su contenido químico y pueden agruparse en dos categorías.

Las cualidades que influyen en el grado de ductilidad del material son elasticidad, maleabilidad, rebote y memoria elástica. Estas características se encuentran pre- 
sentes en grados variables en la mayoría de los materiales termoplásticos e influyen directamente en el esfuerzo requerido por el terapeuta para noldear férulas.

Los materiales altamente moldeables requieren poco esfuerzo del terapeuta para su conformación. Una vez calentados y cortados a medida, estos materiales se amoldan y estiran fácilmente, permitiendo un ajuste continuo con mínima intervención del terapeuta.

\subsection{ORTHOPLAST}

PRESENTACIÓN:

Lámina $61 \times 45 \mathrm{~cm}$

COLOR:

Blanco

TEMPERATURA:

75 a 80 grados

CARACTERÍSTICAS:

Es resistentes, dúctil, maleable, se puede cortar fácilmente aún en seco y frío, pega consigo mismo y con cloruro u otro material de pegado de plásticos, su superficie es lisa y no se muestra pegajoso.

\section{RECOMENDACIONES:}

- Tener cuidado en el manejo para no crear huellas incorrectas en el acabado.

- Debe guardarse en lugar seco y obscuro ya que de otra manera se torna amarillento y pierde sus facultades de maleabilidad.

- Para asearlo se utiliza un cepillo con crema dental y agua fría. 


\subsubsection{POLIURETANO DE DENSIDAD MEDIA}

El poliuretano, piel integral es un polímero termoestable, que se produce por la reacción química entre el poliol y el isocianato. La presencia de $\mathrm{H} 20$ en el proceso hace que se genere $\mathrm{C} 02$, el cual da la estructura celular, para promover y controlar la reacción de impartir propiedades físicas, son necesarias algunos aditivos como espumantes, retardantes a la llama, etc.

Su formula es: $O=C=N-R=C=O(+) H-O-R-H=O-C-N-R-N-C-O-R$

Disocianato + poliol $=$ Poliuretano

\section{PROPIEDADES:}

Gravedad específica: $600-800 \mathrm{gr} / \mathrm{tt}$

Dureza Shore "A" variable $40-90$ pts

Deformación permanente (al 50 ) menor que $70 \%$

Desgarre (4mm) mayor que $300 \mathrm{gr} / \mathrm{mm}$

Carga a la rotura mayor que $40 \mathrm{dm} / \mathrm{cm}^{2}$

Envejecimiento: 72 horas a $110^{\circ} \mathrm{C}$ menos que $30 \%$

\section{CARACTERÍSTICAS:}

Poliuretano piel integral

Material no tóxico

Bajo costo

Bajo peso

Permite manejo de texturas y color

Producción nacional

Se pueden hacer cortes rápidos y fáciles para adaptar a las necesidades requeridas, dar diferentes calibres $(2 \mathrm{~mm}, 3 \mathrm{~mm}, 4 \mathrm{~mm}, 5 \mathrm{~mm}, 1 \mathrm{~cm})$, graduar la dureza del material, pegar fácilmente cińta de cierre, es impermeable. Material no es apto para el desarrollo de hongos y bacterias. 


\section{PREPARACIÓN:}

- Elaboración del molde (en resinas epoxicas)

- Aplicación desmoldante (ceras desmoldeantes)

- Cubicar el volumen de la pieza a desarrollar

$130 \mathrm{~cm}^{3} \times 0.150 \mathrm{gr} / \mathrm{cm}^{3}=118$ gramos

$260 \mathrm{~cm}^{3} \times 0.150 \mathrm{gr} / \mathrm{cm}^{3}=39 \mathrm{gramos}$

- El total de esta operación es el peso de la materia prima a utilizar la proporción del poliol (a) 65 partes, isocianato (b) 35 partes.

- Se prensan los dos componentes .

- Se precalienta el molde a $40^{\circ} \mathrm{C}$.

- Se mezclan y se agitan los dos componentes en un lapso de tiempo de 10 segundos.

- Se hace el vaciado del material y se cierra herméticamente.

- A los 5 minutos se desmolda la pieza final (férula).

\section{ALTERNATIVAS:}

Se comparo el anterior material con los siguientes materiales escogiendo el material del poliuretano de densidad media.

Resinas poliéster: Material muy tóxico en el uso

Difícil moldeo

Económico

Permite dar texturas

Mediano peso

Producción Nacional

Resinas epoxicas: Material no tóxico

Difícil moldeo

Costoso

Permite dar texturas

Mediano peso 
Manejo del color

Producción Nacional

\section{FORMULACIÓN DE LA HIPÓTESIS}

La realización de la férula en otro material diferente a los termoplásticos de baja temperatura (Orthoplas) como es el material de la Férula tipo II (Poliuretano de densidad media), permitirá una disminución en la inconformidad existente en las ciudades de clima caliente, como sucede en la ciudad de San José de Cúcuta., siempre que este tipo de férula disminuya los costos y molestias y mantenga o supere la efectividad en la reducción de síntomas que la producida por las férulas elaboradas con orthoplas.

\section{VARIABLES}

\subsection{Variables independientes}

Férula Tipo I: Elaborada en material de Orthoplas

Férula Tipo II: Elaborada en material de Pouliuretano de densidad media.

\subsection{Variables dependientes}

- Reducción de síntomas

Dolor

Parestesias

- Beneficios

Costos

Durabilidad 


\section{MÉTODO}

\subsection{Características de la población}

La ciudad de San José de Cúcuta se encuentra ubicada en la subregión oriental del departamento del Norte de Santander teniendo una extensión superficial de 1.176 $\mathrm{Km} 2$ que representan el $5.65 \%$ del departammento.

El número de habitantes de San José de Cúcuta según el último censo de 1993 fue de 482.490 habitantes

Se debe considerando su Area Metropolitana que la conforman los municipios de Cúcuta, Villa del Rosario, el Zulia, Los Patios y San Cayetano que tiene una población de acuerdo al último censo de 1993 de 598.341 habitantes de los cuáles 306.808 habitantes son mujeres.

Esta subregión a zona se distingue por su clima cálido, su topografía plana y por predominar las relaciones binacionales.

\subsection{Estadística}

Se tomo como base la estadística elaborada por el Departamento de Terapia Ocupacional del Instituto del Seguro Social de la ciudad de San José de Cúcuta.

Se registraron 70 casos con síndrome de Túnel de Carpio de los cuales un $82 \%$ se registra en mujeres y de estas mujeres un $48 \%$ son amas de casa y el $37.5 \%$ están en edad media.

Razón por la cuál se escogieron ocho (8) pacientes de esta estadística para realizar la muestra y a cuatro de ellas se les adaptó la Férula tipo I (Orthoplas) y las otras cuatro la férula tipo II (Poliuretano de densidad media).

\section{SUJETOS}

Se escogieron ocho (8) pacientes de sexo femenino en edad media ( 30 a 35 años)con ocupación amas de casa y que presentan el estadio temprano del síndrome del 
Túnel del Carpiano a las cuales se les adaptó a cuatro la férula tipo I y a las otras cuatro la férula tipo II.

\section{INSTRUMENTOS}

Los instrumentos que se han usado en el desarrollo del proyecto son:

- Entrevista ocupacional al paciente.

- Pruebas específicas:

Signo de Tinel

Signo de Phalen

- Formatos control evolución de cada paciente. (Anexo \# 1)

- Aparatos:

Férula tipo I: elaborado en orthoplas.

Férula tipo II: elaborada en poliuretano de densidad media.

- Formula de costos

FÉRULA $\mathrm{i}=$ Costo materiales $(\mathbf{i})+$ Costo Mano de obra(t) + Costo herramientas y otros

i: Tipo de férula

$t$ : Tiempo en horas de confección y adaptación

\section{PROCEDIMIENTO}

\subsection{Desarrollo}

- Seleccionar el material: Se escoge el tipo de material a usar. Se trabajara con el Orthoplas por ser este de los termoplásticos más usados en este tipo de férulas y el poliuretano de densidad media por sugerencia del profesional de Diseño Industrial

- Preparar el equipo y herramienta.

- Elaborar la férula con el paciente: Moldeo. 
- Adaptar la férula al paciente.

- Proporcionar instrucciones de usos, cuidado y permanencia de la férula.

- Control a los ocho (8), quince (15), treinta (30) y cuarenta (40) días. Para esto se diseño el formato control de evolución para cada paciente y que se debe ir desarrollando durante todo el periodo de análisis.

- Análisis de costos: se tomo como base una férula de aproximadamente $25 \mathrm{~cm}$ x $10 \mathrm{~cm}$, encontrando el costo para cada tipo de material de acuerdo a la fórmula de costos.

1. i: Férula tipo I (Orthoplas)

t: Tiempo en horas de confección y adaptación: 1 hora

FÉRULA $i=$ Costo materiales $(i)+$ Costo mano de obra $(t)+$ Costo herramientas y otros

\section{FÉRULA I $=\$ 25.000+\$ 20.000+\$ 3.000=48.000$}

2.i: Férula tipo II (poliuretano de densidad media)

t: Tiempo en horas de confección y adaptación: 1:30 horas

FÉRULA $i=$ Costo materiales $(i)+$ Costo de Mano de obra $(t)+$ costo herramientas y otros

\section{FÉRULA II $=\$ 2.300+\$ 30.000+\$ 900=\$ 33.200$}

- Análisis de resultados: para su análisis se hace un cuadro comparativo a los cuarenta días de adaptación. (Anexo \# 2). En el se evalúa cada paciente comparando los síntomas, signos e inconformidades. 


\subsection{Cromograma}

\begin{tabular}{|l|c|c|c|c|c|c|c|c|}
\hline & & Febrero & Marzo & Abril & Mayo & Junio & Julio & Agosto \\
\hline & 1 & 2 & 3 & 4 & 5 & 6 & 7 & 8 \\
\hline Búsqueda bibliografía & & $\mathrm{X}$ & $\mathrm{X}$ & $\mathrm{X}$ & & & & \\
\hline Revisión teoría y marco conceptual & & $\mathrm{X}$ & $\mathrm{X}$ & $\mathrm{X}$ & & & & \\
\hline Planteamiento del problema & & $\mathrm{X}$ & $\mathrm{X}$ & $\mathrm{X}$ & & & & \\
Hipótesis & & $\mathrm{X}$ & $\mathrm{X}$ & $\mathrm{X}$ & & & & \\
Objetivos & & & & $\mathrm{X}$ & & & & \\
\hline Método tipo estudio & & & & $\mathrm{X}$ & $\mathrm{X}$ & & & \\
Seleccionar muestra & & & & & $\mathrm{X}$ & & & \\
\hline Diseño de instrumentos & & & & & $\mathrm{X}$ & $\mathrm{X}$ & & \\
\hline Recolección de datos & & & & & $\mathrm{X}$ & $\mathrm{X}$ & $\mathrm{X}$ & \\
\hline Análisis de resultados & & & & & & & & $\mathrm{X}$ \\
\hline Elaboración de informe final y entrega & & & & & & \\
\hline
\end{tabular}

\section{RESULTADOS}

Después de evaluar durante cuarenta días el desarrollo de la muestra mediante los instrumentos descritos se presentaron los siguientes resultados:

- Todós los pacientes que se le confeccionó y adaptó la férula I (material Orthoplas), tuvieron una disminución entre el $90 \%$ y el $100 \%$ de la sintomatología en cuanto a dolor y parestesias. Con los pacientes que se les adaptó la Férula tipo II (Poliuretano densidad media), la disminución de los síntomas en cuanto dolor y parestesias, fue considerablemente menor, desconocemos si esto se debió a la falta de prática en la realización de férulas con este material.

- Se demostró que el material de poliuretano de densidad media es un $30.83 \%$ más económico que el Ort'.uplas, esto debido a que el poliuretano es un material muy económico, fácil de adaptar a las necesidades requeridas y de cortes rápidos.

- Entre las bondades encontradas en el material de poliuretano de densidad media se destacan el que se puede producir en cualquier color, se pueden 
producir texturas en el material o superficies lisas, dar diferentes calibres, colocar refuerzos e incrustaciones en el material dependiendo de la zona que se quiera inmovilizar, graduar la dureza del material, pegar fácilmente cintas de cierre, es impermeable e inoloro y de acuerdo a la información del material es un material no apto para el desarrollo de hongos y bacterias.

- La férula tipo I en Orthoplas permitió cumplir con los objetivos del tratamiento, siendo de fácil adaptabilidad, sencilla, liviana de rápido arreglo y mantenimiento, y realizar en ella los ajustes según las necesidades particulares del paciente.

- Aunque el material de poliuretano es más económico y presenta beneficios, cabe resaltar que su moldeo puede ser un inconveniente y esto disminuye la efectividad en la mejoría del STC.

- Se concluye que el material Orthoplas que se viene usando aunque es más costoso es el adecuado en la elaboración de férulas en el Síndrome del Túnel del Carpio, porque permite la disminución de los síntomas. Por ello se recomienda continuar el estudio de las férulas elaboradas en poliuretano de densidad media. 

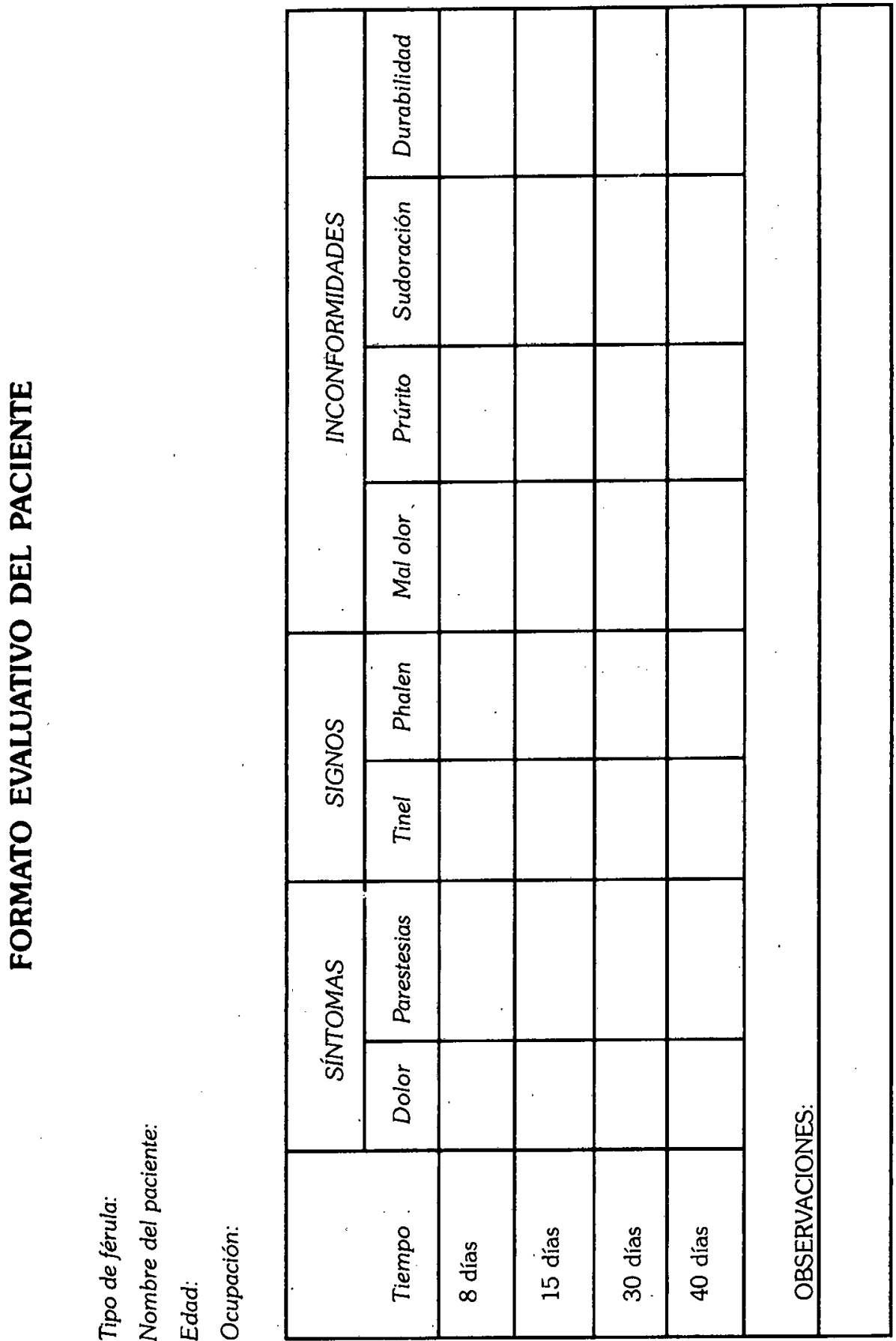

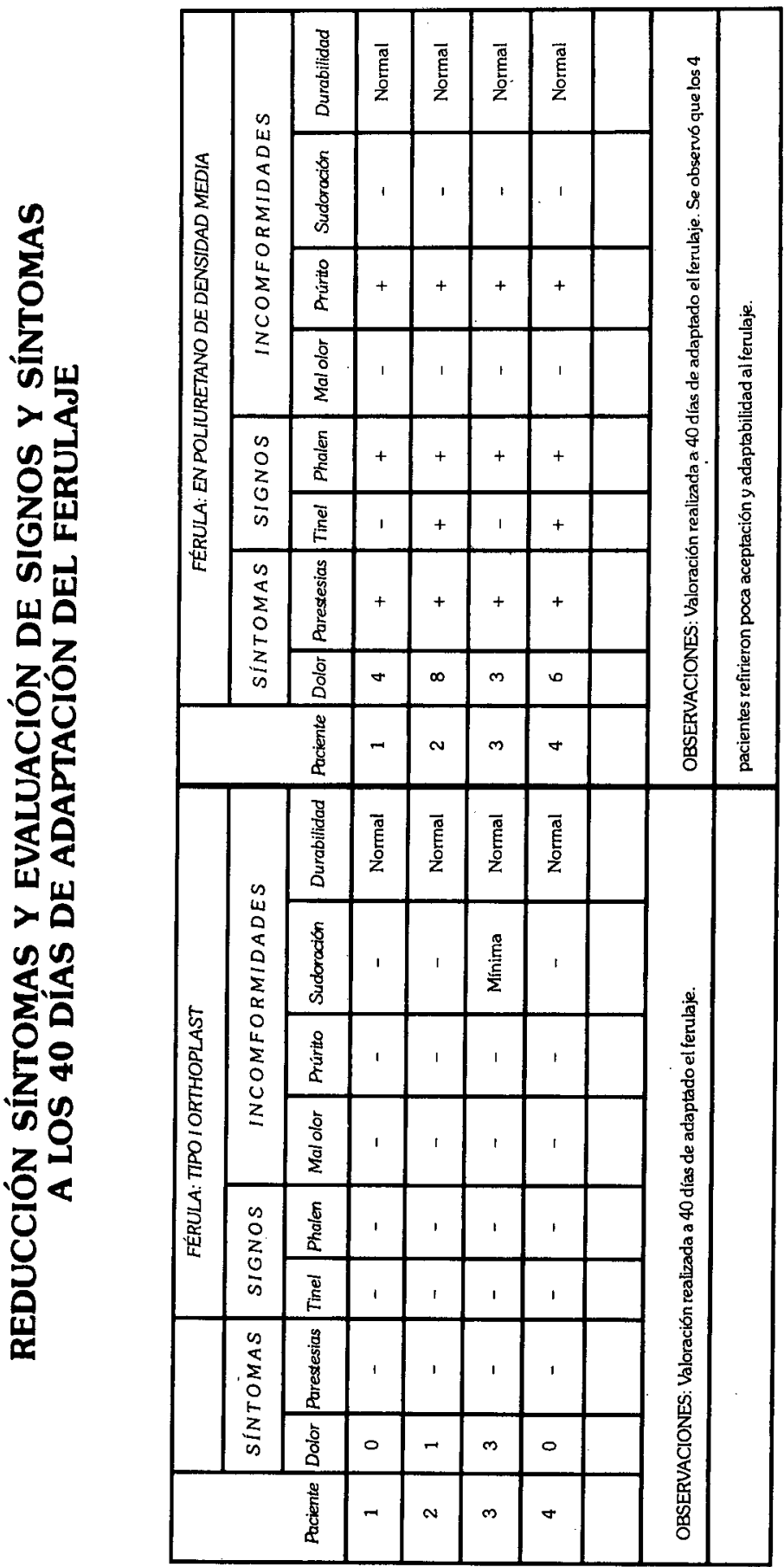


\section{BIBLIOGRAFIA}

McRae, Ronald. Exploración clínica ortopédica. Churchill, Edición Tercera, Madrid, 1993.

Restrepo, Ricardo y Lugo, Luz Elena. Rehabilitación en salud una mirada médica necesaria. Universidad de Antioquía, Primera, Medellín, 1995.

Fotocopias de la Escuela Colombiana de Rehabilitación. Enfoque clínico inicial del paciente con dolor articular, efecto por la manipulación de cargas, posturas inadecuadas y movimientos repetitivos. Efectos por la manipulación.

Páginas WEB, de la revista MED.

Libro de "Mano" 2a. edición, Editorial Manual Moderna Autor, RENE CAILLIET.

Fotocopias tomadas de Mundo Médico, Nov. de 1990, Dra. Jay Tracy.

Terapia Ocupacional - Willard 15 Packman 8 edición.

Ortesis y prótesis del aparato locomotor - Extremidades superior

- R. Viladot. - O. Cohi, - S. Clorell

The Hand, Examination and diagnosis. 3 Edition. American Society for Sugery of the hand.

Fisioterapia de la mano. Cristine A Moron. 1 edición.

Monografía llustrada de Norte de Santander. II Tomo, 1998. 